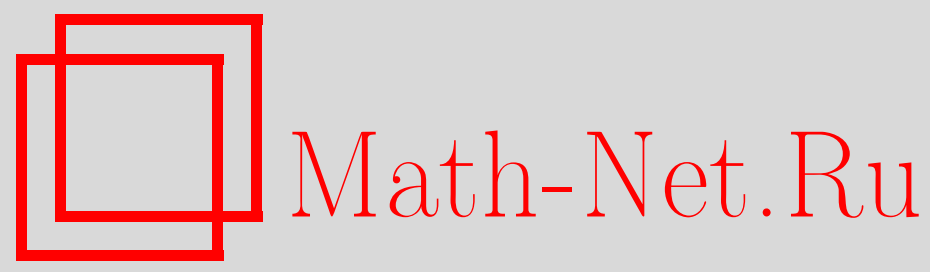

В. 3. Гринес, В. С. Медведев, О топологической сопряженности трехмерных градиентноподобных диффеоморфизмов с тривиально вложенным множеством сепаратрис седловых неподвижных точек, Матем. заметки, 1999, том 66, выпуск 6, 945-948

DOI: https://doi.org/10.4213/mzm1239

Использование Общероссийского математического портала Math-Net.Ru подразумевает, что вы прочитали и согласны с пользовательским соглашением http://www . mathnet.ru/rus/agreement

Параметры загрузки:

IP : 54.84 .234 .179

26 апреля 2023 г., 08:19:12

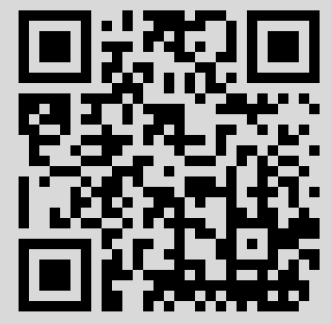




\section{О ТОПОЛОГИЧЕСКОЙ СОПРЯЖЕННОСТИ ТРЕХМЕРНЫХ ГРАДИЕНТНОПОДОБНЫХ ДИФФЕОМОРФИЗМОВ С ТРИВИАЛЬНО ВЛОЖЕННЫМ МНОЖЕСТВОМ СЕПАРАТРИС СЕДЛОВЫХ НЕПОДВИЖНЫХ ТОЧЕК}

\section{В.З. Гринес, В. С. Медведев}

В работах [1], [2] получена топологическая классификация градиентноподобных диффеоморфизмов, а в работах [3]-[5] содержатся необходимые и достаточные условия топологической сопряженности диффеоморфизмов Морса-Смейла с конечным и бесконечньмм множеством гетероклинических траекторий на двумерных многообразиях. Напомним, что диффеоморфизм, заданный на зам-

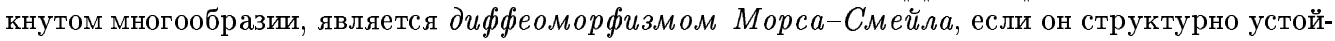
чив и его неблуждающее множество состоит из конечного числа периодических точек. Диффеоморфизм Морса-Смейла называется градиентноподобным, если множество блуждающих точек не содержит гетероклинических траекторий, т.е. устойчивые и неустойчивые сепаратрисы любых седловых периодических точек не пересекаются [6].

В настоящей работе устанавливаются необходимые и достаточные условия топологической сопряженности сохраняющих ориентацию градиентноподобных диффеоморфизмов, заданных на гладком замкнутом ориентируемом трехмерном многообразии в предположении, что неблуждающее множество рассматриваемых диффеоморфизмов состоит из неподвижных точек, а сепаратрисы седловых неподвижных точек удовлетворяют введенному в работе условию тривиальной вложенности. В [7] частично содержатся результаты настоящей работы в случае неприводимого $M$, при этом свойство тривиальной вложенности в этих работах строго не вводится, хотя существенно используется.

Следует отметить, что нетривиальность вложения сепаратрис является принципиально новым моментом, отличающим дискретные динамические системы на трехмерных многообразиях от потоков на них, а также от диффеоморфизмов, заданных на поверхностях. В работе [8] приведен принадлежащий Х. Бонатти пример градиентноподобного диффеоморфизма трехмерной сферы, неблуждающее множество которого состоит из одной седловой, двух стоковых и одной источниковой неподвижных точек, при этом двумерная сепаратриса и одна из одномерных сепаратрис седловой неподвижной точки являются инвариантными дико вложенными двумерной сферой и дугой соответственно (определение дико вложенных объектов см., например, в [9]). Следствием этого является сушествование на трехмерной сфере двух топологически несопряженных градиентноподобных диффеоморизмов, неблуждающие множества которых состоят из одной седловой неподвижной точки с инвариантньми сепаратрисами, двух стоковых и одной источниковой неподвижных точек. Подчеркнем, что в силу [10] два аналогичных потока Морса-Смейла на трехмерной сфере топологически эквивалентны, а в силу [1]-[3] два аналогичных градиентноподобных диффеоморфизма двумерной сферы топологически сопряжены.

Пусть $f: M \rightarrow M$-сохраняющий ориентацию градиентноподобный диффеоморфизм, заданньй на гладком замкнутом ориентируемом трехмерном многообразии $M$. Обозначим через $\Omega(f)$ множество периодических точек диффеоморфизма $f$ и представим множество седловых периодических точек в виде $\Omega_{1}(f) \cup \Omega_{2}(f)$, где $\Omega_{1}(f)\left(\Omega_{2}(f)\right)$ состоит из точек $p$ таких, что $\operatorname{dim}\left(W^{u}(p)\right)=1$ $\left(\operatorname{dim}\left(W^{u}(p)\right)=2\right)$. Будем предполагать, что $\Omega_{1}(f) \cup \Omega_{2}(f) \neq \varnothing^{1}$. Для $\sigma \in\{s, u\}$ положим

$$
W^{\sigma}=\bigcup_{p \in \Omega_{1}(f) \cup \Omega_{2}(f)}\left(W^{\sigma}(p) \backslash p\right), \quad W=W^{s} \cup W^{u} .
$$

Работа выполнена при частичной финансовой поддержке Российского фонда фундаментальнх исследований, грант № 99-01-00230, совместного проекта INTAS-RFBR, грант № 95-418, и фонда INTAS, грант № 97-1843.

${ }^{1}$ Если $\Omega_{1}(f) \cup \Omega_{2}(f)=\varnothing$, то неблуждающее множество диффеоморфизма $f$ состоит в точности из двух неподвижных точек, являющихся источником и стоком. Тогда многообразие $M$ является трехмерной сферой и все такие диффеоморфизмы топологически сопряжены между собой. 
Компоненты связности из множества $W$ обычно называют сепаратрисами (одномерными или двумерными) седловых периодических точек.

В этой работе мы будем предполагать, что все периодические точки данного диффеоморфизма Морса-Смейла $f$ являются неподвижными и все сепаратрисы седловых периодических точек являются инвариантными относительно $f$.

Пусть $\omega \in \Omega(f)$ - стоковая неподвижная точка диффеоморфизма $f$. Обозначим через $L^{u}(\omega)$ множество всех сепаратрис из $W^{u}$, которые содержат $\omega$ в своем замыкании. Множество $L^{u}(\omega)$ состоит из $k_{1} \geqslant 0$ сепаратрис, размерность которых равна 1 , и $k_{2} \geqslant 0$ сепаратрис, размерность которых равна 2. При этом, так как множество седловых периодических точек непусто, одно из чисел $k_{1}, k_{2}$ отлично от 0 . В случае $k_{1} \neq 0$ обозначим через $L_{1}^{1 u}, \ldots, L_{1}^{k_{1} u}$ все одномерные сепаратрисы, а в случае $k_{2} \neq 0$ обозначим через $L_{2}^{1} u, \ldots, L_{2}^{k_{2} u}$ все двумерные сепаратрисы из множества $L^{u}(\omega)$.

Введем в множестве $W^{s}(\omega) \backslash \omega$ соотношение эквивалентности, считая точки $x, y \in W^{s}(\omega) \backslash \omega$ экивалентными, если существует $n \in \mathbb{Z}$ такое, что $y=f^{n}(x)$. Группа преобразований $F$, порожденная диффеоморфизмом $f$, является дискретной группой преобразований, свободно действующей на множестве $W^{s}(\omega) \backslash \omega$. Поэтому факторпространство $N_{\omega}=\left(W^{s}(\omega) \backslash \omega\right) / F$ по введенному соотношению эквивалентности является хаусдорфовым пространством, которое можно снабдить топологией гладкого многообразия. Обозначим через $\pi \omega$ естественную проекцию множества $W^{s}(\omega) \backslash \omega$ на многообразие $N_{\omega}$. Так как сток $\omega$ является гиперболической неподвижной точкой, существует гладкое вложение $S_{E}$ стандартной двумерной сферы $S^{2}: x_{1}^{2}+x_{2}^{2}+x_{3}^{2}=1\left(x_{1}, x_{2}, x_{3}\right.$ - декартовы координаты в трехмерном евклидовом пространстве) такое, что $S_{E}$ является границей открытого трехмерного диска $D_{\omega}$, содержащего сток $\omega$, и поверхность $S_{I}=f\left(S_{E}\right)$ целиком принадлежит $D_{\omega}$. Тогда замыкание области $R(\omega)$, ограниченной поверхностями $S_{E}, S_{I}$, является фундаментальной областью группы $F$. Отсюда следует, что многообразие $N_{\omega}$ гомеоморфно произведению $S^{2} \times S^{1}$ посредством некоторого гомеоморфизма $\psi: N_{\omega} \rightarrow S^{2} \times S^{1}$. Универсальным накрытием для $S^{2} \times S^{1}$ является произведение $S^{2} \times \mathbb{R}$. Обозначим через $\pi$ естественную проекцию $S^{2} \times \mathbb{R}$ на $S^{2} \times S^{1}$ и через $T$ движение на пространстве $S^{2} \times \mathbb{R}$, заданное формулой $T(s, t)=(s, t+1)$, где $s \in S^{2}, t \in \mathbb{R}$. Тогда существует накрывающее отображение $\bar{\psi}: W^{s}(\omega) \backslash \omega \rightarrow S^{2} \times \mathbb{R}\left(\pi \bar{\psi}=\psi \pi_{\omega}\right)$ такое, что $\bar{\psi} f=T \bar{\psi}$. Положим

$$
\gamma_{1}^{1}=\pi_{\omega}\left(L_{1}^{1 u}\right), \quad \ldots, \quad \gamma_{1}^{k_{1}}=\pi_{\omega}\left(L_{1}^{k_{1} u}\right), \quad \gamma_{2}^{1}=\pi_{\omega}\left(L_{2}^{1 u}\right), \quad \ldots, \quad \gamma_{2}^{k_{2}}=\pi_{\omega}\left(L_{2}^{k_{2} u}\right) .
$$

Множество $\gamma_{1}^{i}\left(i \in\left\{1, \ldots, k_{1}\right\}\right)$ является гладким вложением в $N_{\omega}$ стандартной окружности. Так как диффеоморфизм $f$ сохраняет ориентацию многообразия $M$ и каждая одномерная сепаратриса седловой неподвижной точки является инвариантной относительно $f$, ограничение диффеоморфизма $f$ на двумерную сепаратрису каждой седловой неподвижной точки также является сохраняющим ориентацию отображением. Поэтому множество $\gamma_{2}^{j}\left(j \in\left\{1, \ldots, k_{2}\right\}\right)$ является гладким вложением стандартного двумерного тора.

ОПРЕДЕЛЕНИЕ 1. Множество

$$
S(\omega)=\left(\bigcup_{i \in\left\{1, \ldots, k_{1}\right\}} \gamma_{1}^{i}\right) \cup\left(\bigcup_{j \in\left\{1, \ldots, k_{2}\right\}} \gamma_{2}^{j}\right)
$$

назовем схемой стоковой неподвижной точки $\omega$.

Зададим на двумерной сфере $S^{2} \times\{0\} k_{1} \geqslant 0$ различных точек $s_{1}, \ldots, s_{k_{1}}$ и $k_{2} \geqslant 0$ простых не пересекающихся между собой замкнутых кривых $c_{1}, \ldots, c_{k_{2}}$, каждая из которых не проходит ни через одну из точек $s_{1}, \ldots, s_{k_{1}}\left(k_{1}^{2}+k_{2}^{2} \neq 0\right)$. Положим $\nu_{1}^{i}=\pi\left(s_{i} \times \mathbb{R}\right), \nu_{2}^{j}=\pi\left(c_{j} \times \mathbb{R}\right)$ $\left(i \in\left\{1, \ldots, k_{1}\right\}, j \in\left\{1, \ldots, k_{2}\right\}\right)$. По построению множество $\nu_{1}^{i}$ является гладким вложением стандартной окружности, а $\nu_{2}^{j}$ является гладким вложением стандартного двумерного тора, и множества $\nu_{r}^{i}, \nu_{l}^{j}$ не пересекаются, если выполняется хотя бы одно из условий $i \neq j, r \neq l$. 
ОПРЕДЕЛЕНИЕ 2. Объединение

$$
L\left(k_{1}, k_{2}\right)=\left(\bigcup_{i \in\left\{1, \ldots, k_{1}\right\}} \nu_{1}^{i}\right) \cup\left(\bigcup_{j \in\left\{1, \ldots, k_{2}\right\}} \nu_{2}^{j}\right), \quad k_{1}^{2}+k_{2}^{2} \neq 0
$$

назовем тривиальным зацеплением в $S^{2} \times S^{1}$. При этом если $k_{1}=0\left(k_{2}=0\right)$, то тривиальное зацепление не содержит вложений окружности (тора).

ОПРЕДЕЛЕНИЕ 3. Два тривиальных зацепления

$$
\begin{aligned}
L\left(k_{1}, k_{2}\right) & =\left(\bigcup_{i \in\left\{1, \ldots, k_{1}\right\}} \nu_{1}^{i}\right) \cup\left(\underset{j \in\left\{1, \ldots, k_{2}\right\}}{\bigcup} \nu_{2}^{j}\right), \\
L^{\prime}\left(k_{1}^{\prime}, k_{2}^{\prime}\right) & =\left(\bigcup_{i \in\left\{1, \ldots, k_{1}^{\prime}\right\}} \nu_{1}^{\prime i}\right) \cup\left(\bigcup_{j \in\left\{1, \ldots, k_{2}^{\prime}\right\}} \nu_{2}^{\prime j}\right)
\end{aligned}
$$

назовем изоморфными, если $k_{1}=k_{1}^{\prime}, k_{2}=k_{2}^{\prime}$ и существует гомеоморфизм $\varphi: S^{2} \times S^{1} \rightarrow S^{2} \times S^{1}$ такой, что $\varphi\left(\nu_{1}^{i}\right)=\nu_{1}^{\prime i}, \varphi\left(\nu_{2}^{j}\right)=\nu_{2}^{\prime j}\left(i=\overline{1, k_{1}}, j=\overline{1, k_{2}}\right)$.

ОПРЕДЕЛЕНИЕ 4. Будем говорить, что множество сепаратрис $L^{u}(\omega)$ тривиально вложено, если существует тривиальное зацепление

$$
L\left(k_{1}, k_{2}\right)=\left(\bigcup_{i \in\left\{1, \ldots, k_{1}\right\}} \nu_{1}^{i}\right) \cup\left(\bigcup_{j \in\left\{1, \ldots, k_{2}\right\}} \nu_{2}^{j}\right)
$$

и гомеоморфизм $\psi: N_{\omega} \rightarrow S^{2} \times S^{1}$ такие, что $\psi\left(\gamma_{1}^{i}\right)=\nu_{1}^{i}, \psi\left(\gamma_{2}^{j}\right)=\nu_{2}^{j}\left(i=\overline{1, k_{1}}, j=\overline{1, k_{2}}\right)$. В этом случае будем говорить, что схема стоковой неподвижной точки $\omega$ тривиальна.

Зацепление $L\left(k_{1}, k_{2}\right)$ будем назьвать соответств ующим схеме неподвижной точки $\omega$.

ОПРЕДЕЛЕниЕ 5 . Ориентированный граф $G(f)$ назовем графом диффеоморфизма $f$, если вершины графа $G(f)$ соответствуют неподвижным точкам из множества $\Omega(f)$, а его ребра соответствуют компонентам связности из множеств $W$.

При этом ориентация на любом ребре $l^{\sigma}$ задается следующим образом. Если ребру $l^{\sigma}$ инцидентны вершины $a$ и $b$ и $l^{\sigma}$ отвечает сепаратрисе $L^{\sigma}(p)$ седловой неподвижной точки $p$, то одна из вершин, например $a$, отвечает точке $p$, а вершина $b$ отвечает либо источнику (если $\sigma=s$ ), либо стоку (если $\sigma=u)$. Тогда ориентация на ребре $l^{\sigma}$ выбирается от $b$ к $a$, если $\sigma=s$, и от $a$ к $b$, если $\sigma=u$.

Пусть $f, f^{\prime}: M \rightarrow M$ - два сохраняющих ориентацию градиентноподобных диффеоморфизма и $\omega, \omega^{\prime}$ - стоковые неподвижные точки $f, f^{\prime}$ соответственно, обладающие тривиальными схемами $\omega, \omega^{\prime}$.

ОПРЕДЕЛЕНИЕ 6. Назовем тривиальные схемы неподвижных точек $\omega, \omega^{\prime}$ эквивалентными, если тривиальные зацепления $L\left(k_{1}, k_{2}\right), L^{\prime}\left(k_{1}, k_{2}\right)$, соответствующие точкам $\omega, \omega^{\prime}$, изоморфны.

ОПРЕДЕЛЕНИЕ 7. Будем говорить, что градиентноподобный диффеоморфизм $f$ принадлежит классу $G(M)$, если он удовлетворяет следующим условиям:

1) $f \in G(M)$ сохраняет ориентацию многообразия $M$;

2) неблуждающее множество диффеоморфизма $f \in G(M)$ состоит из неподвижных точек, и все сепаратрисы седловых неподвижных точек инвариантны относительно $f$;

$3)$ для каждой стоковой неподвижной точки $\omega \in \Omega(f)$ множество $L^{u}(\omega)$ всех сепаратрис, содержащих $\omega$ в своем замыкании, тривиально вложено (см. определение 4$)$. 
ОПРЕДЕЛЕНИЕ 8. Графы $G(f), G^{\prime}\left(f^{\prime}\right)$ диффеоморфизмов $f, f^{\prime} \in G(M)$ назовем изоморфнымu, если существует сохраняющий ориентацию ребер изоморфизм $\eta$ графов $G(f), G^{\prime}\left(f^{\prime}\right)$ такой, что выполняются следуюшие условия:

1) изоморффые в силу $\eta$ вершины граффов $G(f), G^{\prime}\left(f^{\prime}\right)$ отвечают топологически эквивалентньпм неподвижнып точкам диффеоморфизмов $f$ и $f^{\prime}$;

2) если изоморфные в силу $\eta$ вершины графов соответствуют стоковым неподвижньм точкам диффеоморфизмов $f$ и $f^{\prime}$, то их схемы эквивалентны.

Теорема 1. Пусть $f, f^{\prime} \in G(M)$. Тогда, для того чтобы существовал сохраняющий ориентацию гомеоморфизм $g: M \rightarrow M$ такой, что $f^{\prime}=g g^{-1}$, необходимо и достаточно, чтобь графы $G^{*}(f)$ и $G^{*}\left(f^{\prime}\right)$ били изоморфны.

\section{СПИСОК ЦИТИРОВАННОЙ ЛИТЕРАТУРЫ}

1. Безденежных А. Н., Гринес В.З. // Методы качественной теории дифференциальных уравнений / ред. Е. А. Лентович-Андронова. Межвуз. темат. сб. научн. тр. Горький: ГГУ, 1985. С. 22-38; 1987. С. 24-32. 2. Безденежных А. Н., Гринес В. З. // Дифференциальные и интегральные уравнения / ред. Н. Ф. Отроков. Сб. научн. тр. Горький: ГГУ, 1985. С. 33-37. 3. Гринес В. 3. // Матем. заметки. 1993. Т. 54. №3. С. 3-17. 4. Langevin R. // Ann. Inst. Fourier (Grenoble). 1993. V. 43. № 1. P. 265-278. 5. Bonatti C., Langevin R. Diffeomorphismes de Smale des surfaces. Astérisque. V. 250. Montrouge: Soc. Math. France, 1998. 6. Смейл С. // УМН. 1970. Т. 25. № 1. С. 113-185. 7. Гринес В. З., Калай Х. Х. // УМН. 1994. Т. 49. № 2. С. $149-150$. 8. Bonatti Ch., Grines V.Z. Knots as topological invariant for gradient-like diffeomorphisms of the sphere $S^{3}$ (to appear). 9. Келдыш Л. В. // Тр. МИАН. 1966. Т. 81. С. 1-183. 10. Уманский Я. Л. // Матем. сб. 1990. Т. 181. № 2. С. 212-239. 